\title{
Biomimetics as problem-solving, creativity and innovation tool in a first year engineering design and communication course
}

\author{
M. J. Eggermont \\ Schulich School of Engineering, University of Calgary, Canada
}

\begin{abstract}
Engineering sketching, as taught in our first-year design course, exists somewhere between writing and formal drawing as a means of formulating ideas. In our third year of teaching engineering sketching assignments were given several additional components: the visualization of engineering concepts, sustainable product design and biomimetics. This was done for a number of reasons: Students were given the opportunity to integrate knowledge from other first year engineering courses; Students who were not necessarily strong artists felt they could make up for this in the 'additional component' category; First year students seem to have a great interest in the study of structural biology as it applies to engineering design. Now in our sixth year, this paper discusses biomimetics, the abstraction of good design from nature or the transfer of technological ideas from nature to artificial applications and the resulting student projects. In our current political environment of sustainable design and sustainable design solutions (even 'ecofriendly fire'), biomimetics offers an interesting approach to design. This multidisciplinary approach impresses upon novice designers the importance of teamwork; it also explores a topic that lends itself easily to be co-taught across faculties. Students use engineering sketching and the latest biomimetic research to come up with solutions for everyday activities, objects and environments. This paper will also discuss some of the many designs that have resulted from these projects.
\end{abstract}

Keywords: biomimetics, engineering design, problem-solving, innovation, creativity, drawing. 


\section{Introduction}

The Boyer Commission's Report of 1998 called for “a restructuring of undergraduate education at large research institutions. The report emphasized interdisciplinarity, undergraduate participation in research, and the integration of communication skills into course work across the curriculum. At the University of Calgary the Undergraduate Curriculum Redesign Team took up the recommendations of the Boyer Report. The team's final report, released in May 2003, called for a hands-on, inquiry-based approach to learning, particularly for first year students. The Canadian Engineering Accreditation Board (CEAB) stipulates that every student must have real world, team-oriented, open-ended design experience before graduation (CEAB, 2003)."

Engineering 251/253 (ENGG 251/253), a collaboration of art, engineering, and communication, is an interdisciplinary first year engineering design course first implemented in the 2002/2003 academic year in response to the challenge to innovate. Now, in our sixth year, the course is still built around four pillars: drawing, design, communication, and teamwork. All 700 to 750 students entering the engineering faculty participate in the course for a full year.

Students are involved in the performance of drawing as a means for developing the mind/hand/eye coordination necessary for achieving a high level of visual literacy. Technical drawing components are developed as supporting elements to the evolution of ideas, rather than as an end in themselves. In addition to paraline and perspective-based projections, the students are also exposed to design concepts such as the golden section, form-follows-function, and biomimetics. In the last four years, the latter has become a permanent fixture in our design course and will become one of the main projects in 2008/09 (a sixweek biomimetics and robotics project).

This paper will discuss the development of the biomimetic component and show students examples. The student assignments began with external mimicry to a recent study on how students (mis)understand abstraction. In hindsight it seems the projects followed Julian Vincent's links between biology and engineering (Fig. 1).

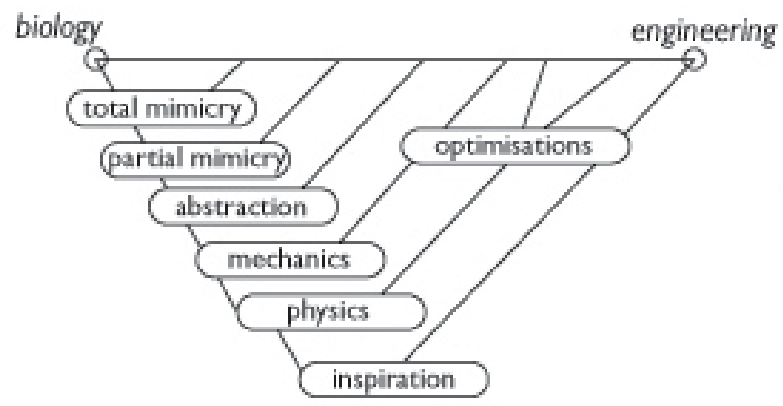

Figure 1: $\quad$ From Julian Vincent's Stealing Ideas from nature [1]. 


\section{Looking back: "Go to the ant, thou sluggard"}

Biomimetics is introduced starting with historical examples such as the Crystal Palace, barbed wire (Fig. 2), Velcro and ends with more recent examples such as spider silk produced in goat's milk, Lotusan paint, the Beijing Olympic Swim stadium, termite-mound-inspired architecture, and Gecko tape.

An introduction to biomimetics and the discussion of research areas currently expanding in this field can generate a lot of enthusiasm among first year students. The biological aspects turn engineering concepts into more tangible examples. The students also feel this is an area of growth and potential new discoveries. In the current climate of sustainable design, biomimetics is an area that promises real change. Sustainable design is easily explained when using biomimetics as teaching model. This area also invites a great deal of creativity. Students have to observe, process, analyze and synthesize different types of information. For the seventeen and eighteen-year-old students this often comes as a shock. The bigger shock (for them) is that they are actually quite good at it.
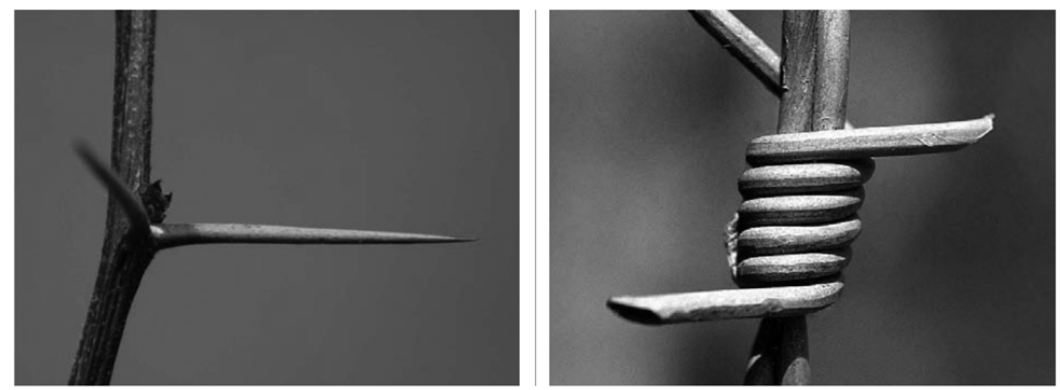

Functional abstraction: thorn to barbed wire

Barbed wire is both a functional and a visual abstraction.

Figure 2: $\quad$ Introduction slide example.

\subsection{A fuzzy creature that also happens to cut things in half}

The first biomimetic assignment was completed in 2003/2004. It required external observation of organisms and required some mechanical abstraction:

"Design a biomimetic hand tool that executes one of the following actions: separate/sort/mix/dig/fasten/join/aid movement. 
Design a tool with a new function (a reasonable and potentially workable function) or redesign an existing tool. The following questions are things to think about:

- What problem are you trying to solve?

- What kind of tool are you thinking about?

- What does the tool do?

- Which natural systems or organisms solve your problem?

- How does nature deal with the natural laws that concern your 'problem'?

- Find as many biological examples as you can that deal with your problem, then choose the one that will help you most.

Please avoid a physical likeness to your source: your drawing should look like a tool, not like a fuzzy creature with ears and a tail that also happens to cut things in half.'

Students focussed largely on sharks and other animals that exhibit dramatic movements. A few focussed on internal, but still largely, observable mechanisms such as the joint capsule example below (Fig. 3).

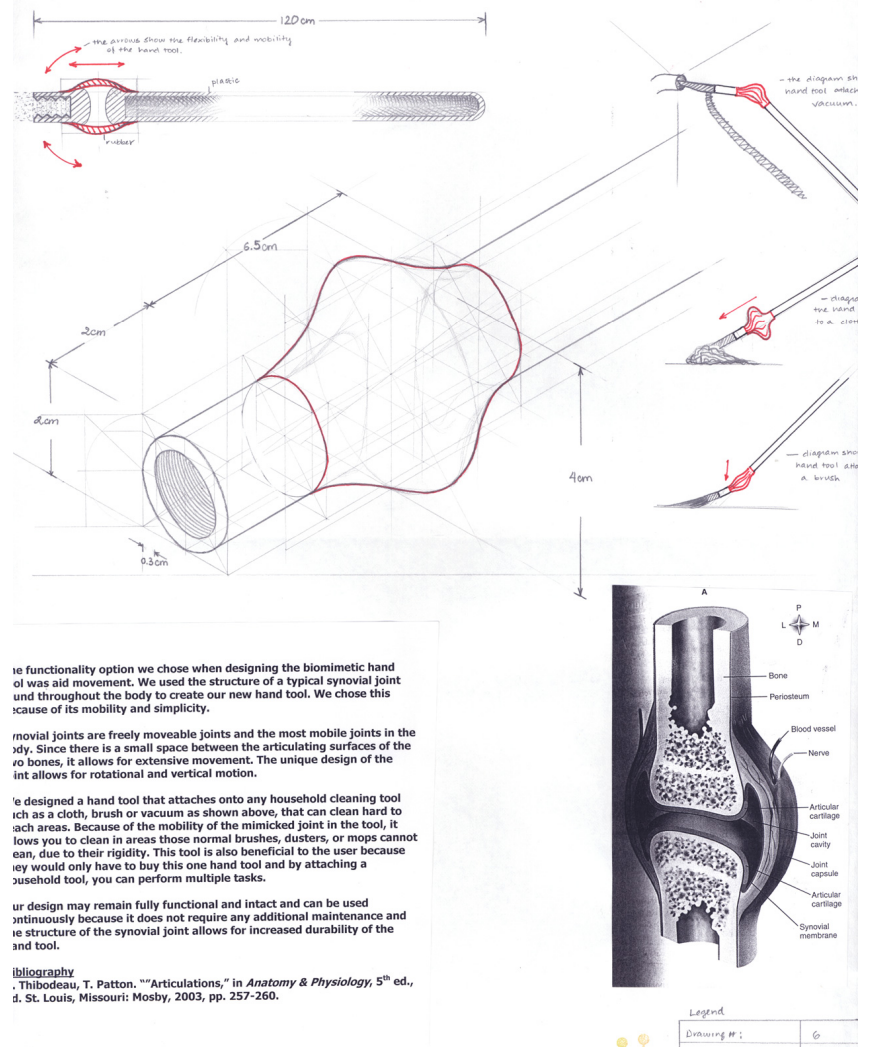

Figure 3: Student drawing: 'Aiding movement'. 


\subsection{Insects and sustainability}

In $04 / 05$ the assignment became more focussed. Students were asked to research insect behaviour and/or locomotion and were asked to improve or redesign a device focussing on sustainable solutions (Fig. 4).

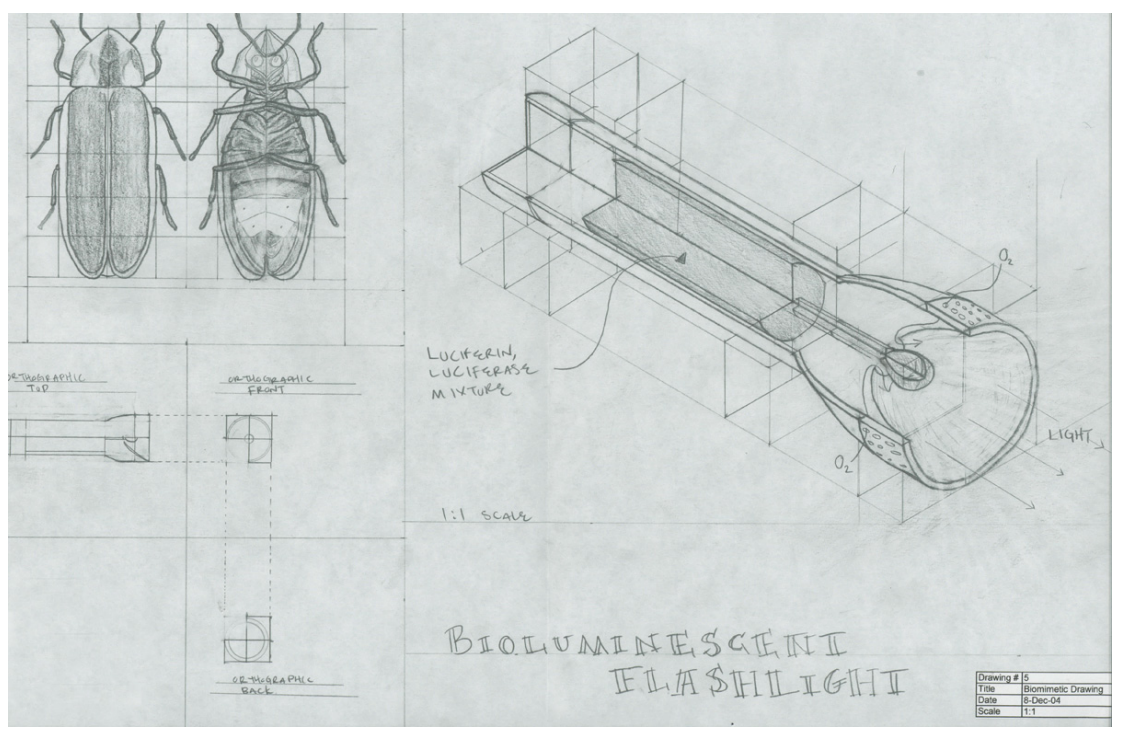

Figure 4: Student drawing: insect behaviour.

\section{3 "Machines are an effect of art, which is nature's ape, and they reproduce not its forms but the operation itself" [2]}

The following year using biomimetics, students designed a structure that was primarily tension-based. They were asked to describe the biological phenomenon the design was based upon, and, using their first year engineering knowledge, describe the process of abstraction that led to their final design (Fig. 5).

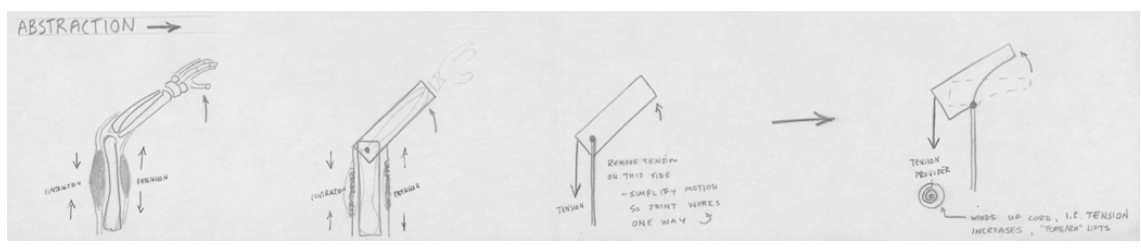

Figure 5: $\quad$ Student drawing: Process of abstraction.

\subsection{Design portal}

In 2006/07 students used scientific research accessed via the Design Portal on the website of the Biomimicry Institute to design solutions or alternative ideas for their apartment or house (Fig. 6). The design ideas could either come from the 
Design Portal or from their own house. The latter scenario required students to look up research that most reflected the problem they were trying to solve or area they were truing to redesigning. The portal divided phenomena in three separate categories: pattern, process and system. This division, in hindsight, triggered the author to look closer at the process of abstraction throughout the students' design process.
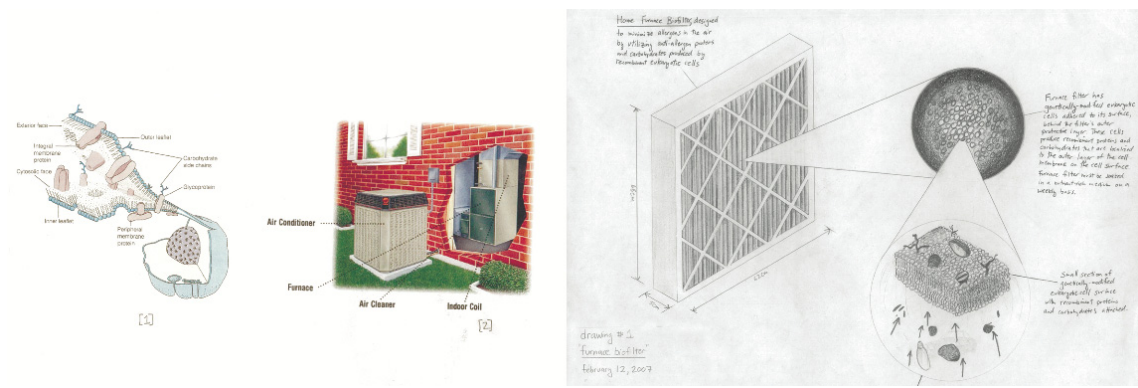

Figure 6: $\quad$ Student drawing: furnace bio-filter.

\subsection{Abstraction}

\subsubsection{No more fuzzy creatures that also happens to cut things in half}

This past year the biomimetics project coincided with a Solar Decathlon project the North-American solar house competition. Students were asked to come up with ideas for the Solar Decathlon team in eight areas of research. Biomimetics was one of those areas. The assignment was as follows:

"Create a design abstracted from on one organism you choose from the groupings assigned to your lab.

Lab A: Echinoderms, arthropods and molluscs

Lab B: Reptiles, sharks and bony fishes

Lab C: Insects, birds and plants

Lab D: Mammals and amphibians

Your abstraction has to be based on 2 out of the 3 abstractions discussed (visual, functional and process). Your abstraction can be a (or part of) building, mechanism or process.

1. Describe your idea in one paragraph.

2. Draw your abstraction in isometric.

3. Create a graph (*see graph below); place your idea and find other examples to complete the graph [This idea was a variation on (3)]. Choose one of 3 graphs below based on your initial choice (Fig. 7)."
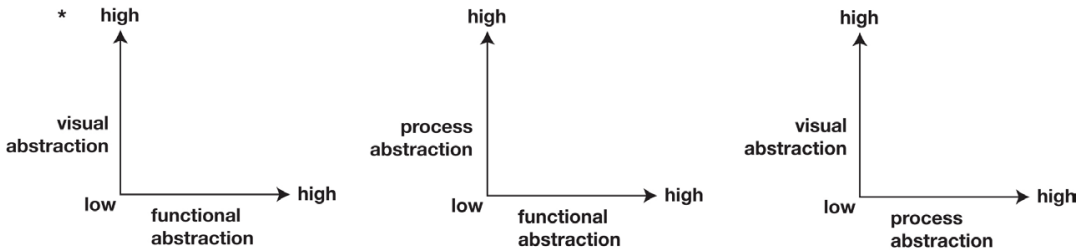


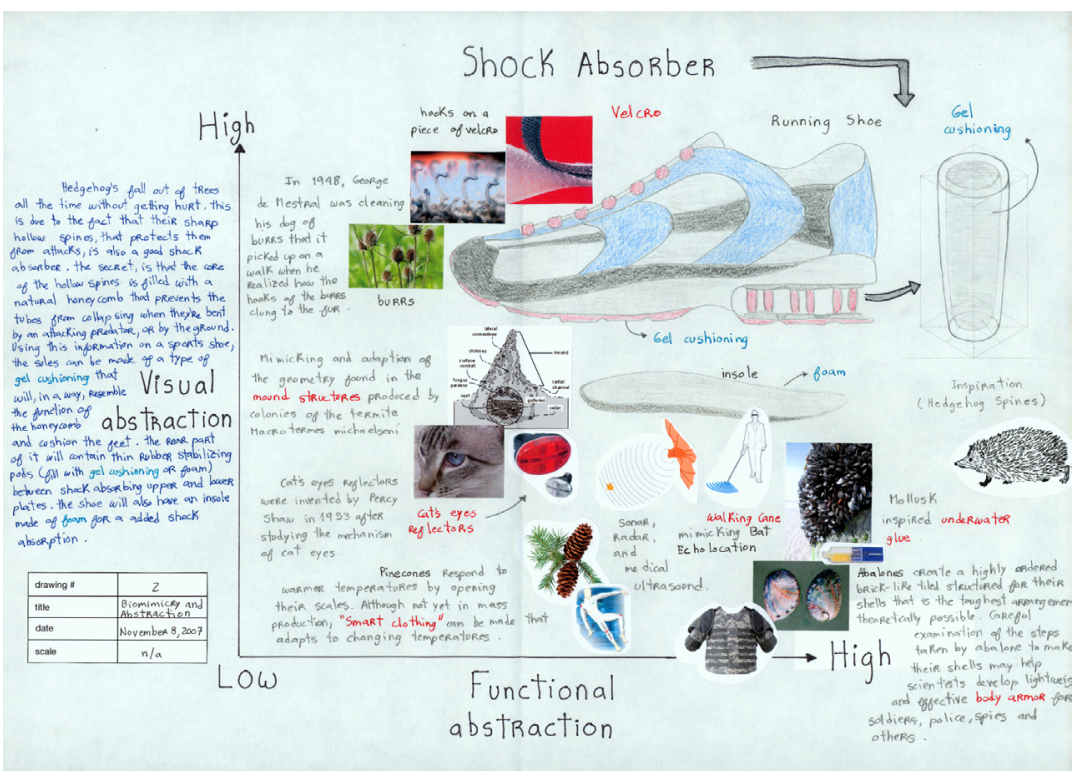

Figure 7: Visual/functional abstraction.

The addition of the graph made a big difference in the outcome of the assignment. Students were first of all required to do more thorough research because the graph had to be filled with many examples. They also had to place their own design idea among existing ideas, which made them think about the 'goodness' of their design: How would it stand up in the real world?

\subsection{2 'Creaturely gestures' versus abstraction}

Peter Forbes in The Gecko's Foot discusses bio-inspired design as it coincides with the general Zeitgeist of organicism. He describes a number of architectural structures that feature "creaturely gestures rather than mimicking specific creatures" [4]. Forbes mentions buildings designed by Santiago Calatrava, known for his abstractions of birds in flight and aspects of the human body.
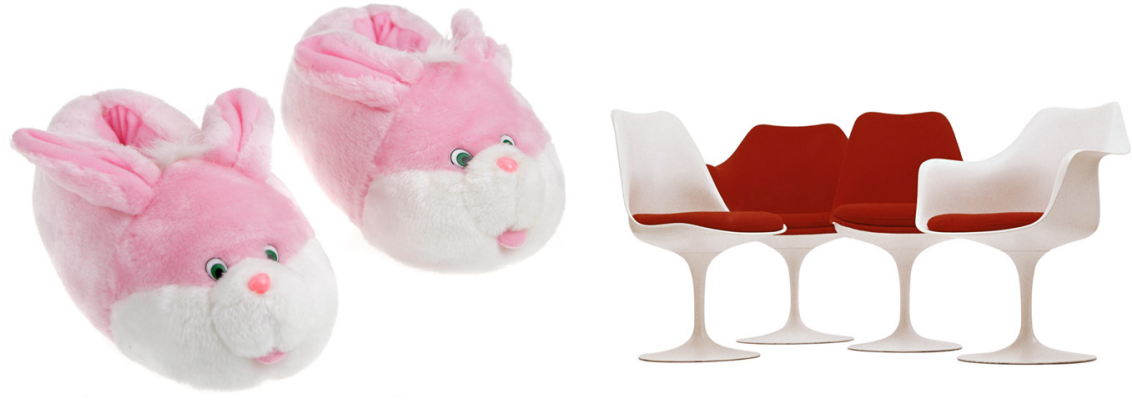

Figure 8: $\quad$ Bunny slippers and Tulip chairs. 
When discussing his Turning Torso apartment building in Malmo this gesture has become known as 'high' visual abstraction and 'low' functional abstraction. An example of 'low' and 'medium' visual abstraction in this system would be bunny slippers and the Tulip Chair by Eero Saarinen (Fig. 8).

\subsubsection{The process of abstraction to test engineering requirements}

Thinking about different forms of abstraction also allowed the students to start thinking about engineering requirements as introduced in the second half of the course. We had our students look at an Inclusive Design Toolkit [7], which described 'good requirements' as follows:

a. Solution independent

b. Specific, objective, and quantified where possible

c. Measurable and testable

d. Traceable so that the source of the requirement is understood

e. Accurate in their representation of the true needs

f. Complete such that any solution that meets the requirements will be satisfactory

g. Well structured

First year engineering students are under the mistaken impression that to be inspired by biology the engineering object that is being created should look like the original organism. Students have difficulty distilling the function out of anything at the best of times. The question: "What does your design do?" seems to be the hardest question we ask each year. Ultimately abstraction is the distillation of the essence, of the pattern (visual and/or functional) of the biological organism. Encouraging the students to distinguish between high/low visual, functional, and process abstraction allowed them to get closer to the first item on the requirements list: a solution independent design (Fig. 9).

The Biomimicry Institute has a design process, the Design Spiral, which focuses on functionality in their Identify stage: "Identify the function you want

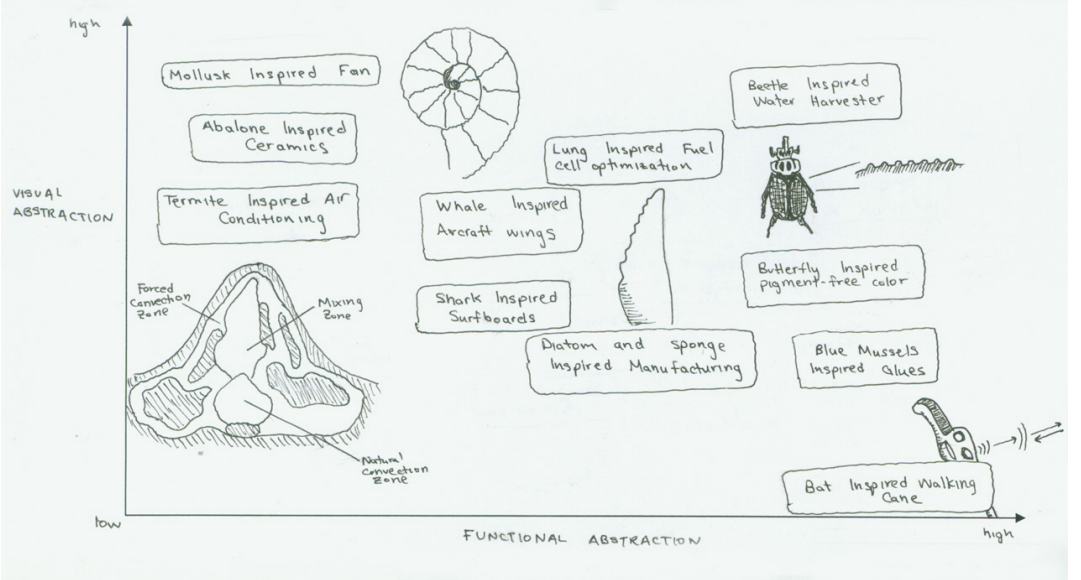

Figure 9: Visual/functional abstraction. 
your design to accomplish: What do you want your design to do? (not 'what do you want to design?'). Continue to ask why until you get to the bottom of the problem"[8]. The abstraction process described previously is very similar, but perhaps a necessary addition for inexperienced engineering student designers.

\section{Conclusion}

\section{1 "I managed to stay as far away from art as I could in High School...that quest ended when I started first year Engineering Design and Communication." - Student quote}

In our course technical drawing components are developed as supporting elements to the evolution of ideas, rather than as an end in themselves. Biomimetics has become a permanent feature in our course. We explore its history and current research areas. Biomimetics allows students a lot of freedom in their design, but also links engineering concepts to tangible examples. To date, over 2500 biomimetics drawings have been created.

One of the key features of a design course is that students study subjects in breadth rather than depth. In studying a wide variety of subjects, design courses employ a wider variety of specialists and this facilitates a cross-linking of cultures and perspectives. The conjoining of previously unrelated ideas, thoughts and concepts is well recognized as a feature of creative thinking. Introducing a full range of subjects allows students to practice and develop their integrative skills [9]. Biomimetics is integrative by nature.

\section{References}

[1] Pellegrino, S., Deployable Structures, Springer-Verlag: Berlin and New York, pp. 51-58, 2002.

[2] Eco, U., The Name of the Rose, Harcourt Brace Jovanovich: New York, p. $1-7,1983$.

[3] Vincent, J., and Mann, D., Systematic technology transfer from biology to engineering.

[4] Forbes, P., The Gecko's Foot, Harper Perennial: New York, p. 27, 2005.

[5] Bunny slippers, http://images.netshops.com/mgen/master:NIX036.jpg [accessed Feb. 23, 2008]

[6] Tulip chairs, http://upload.wikimedia.org/wikipedia/en/e/e7/Tulip_med.jpg [accessed Feb. 23, 2008]

[7] Clarkson, J., Coleman, R., Hosking, I., Waller, S., Inclusive Design Toolkit, Kall Kwik: Cambridge, p. 2-42, 2007.

[8] Biomimicry Institute, http:/www.biomimicryinstitute.org/about-us/ biomimicry-a-tool-for-innovation.html [accessed Feb. 23, 2008].

[9] Morris, R., Childs, P., and Hamilton, T., Sustainability by design: a reflection on the suitability of pedagogic practice in design and engineering courses in the teaching of sustainable design. European Journal of Engineering Education, 32:2, 135-142, 2007. 